
\title{
$\angle$ Research Square \\ The Utilization of Warfarin Dosing Algorithms in China: An Electronic Survey Study
}

\section{Yaxin Liu}

Central South University Xiangya School of Pharmaceutical Science

\section{Tian Wu}

Central South University Third Xiangya Hospital

\section{Yun Kuang}

Central South University Third Xiangya Hospital

\section{Xiaoyi Ning}

Central South University ,the third Xiangya hospital

\section{Jinlian Xie}

Central South University, the third Xiangya hospital

\section{Chengxian Guo}

Central South University Third Xiangya Hospital

\section{Yuxia Xiang}

Central South University Third Xiangya Hospital

\section{Weihong Jiang}

Central South University Third Xiangya Hospital Department of Cardiology

Guoping Yang ( ygp9880@126.com )

XiangYa School of Pharmaceutical Sciences, Central South University, Changsha, Hunan, 410013, China

\section{Research}

Keywords: Warfarin dosing algorithms, Chinese medical professionals, Online questionnaire

Posted Date: December 31st, 2020

DOl: https://doi.org/10.21203/rs.3.rs-134986/v1

License: (c) (i) This work is licensed under a Creative Commons Attribution 4.0 International License.

Read Full License 


\section{Abstract}

Background: Warfarin, a key anticoagulant medication, has a narrow therapeutic window and individual difference. Many warfarin dosing algorithms have been developed and been proved to have clinical benefits. However, the utilization of algorithms by medical professionals in China was unknown. We conducted an online survey to investigate the use and requirements of warfarin dosing algorithms among Chinese medical professionals.

Method: A questionnaire survey was conducted via WeChat to investigate the utilization of warfarin dosing algorithms by medical professionals in seven regions of China. The content of questionnaire was included general characteristics of participants, condition of anticoagulant therapy, utilization of warfarin dosing algorithms and demand for function of an assistant warfarin dosing system we proposed.

Results: A total of 399 participants completed the survey. Although most medical professionals use warfarin for anticoagulant therapy, some of them (15.04\%) were not familiar with warfarin's individual difference. As high as $32.97 \%$ of clinicians ruled out genotypes when using warfarin. The vast majority of anticoagulant medical professionals believed warfarin dosing algorithms can have clinical benefits, but only $20.80 \%$ of them usually use algorithms for anticoagulant therapy.

Conclusion: Warfarin dosing algorithms have high evaluation while not good utilization among Chinese anticoagulant medical professionals. If warfarin dosing algorithms and assistant tools aimed to Chinese population were developed, to some extent can improve anticoagulant therapy in China.

\section{Full Text}

This preprint is available for download as a PDF.

\section{Figures}
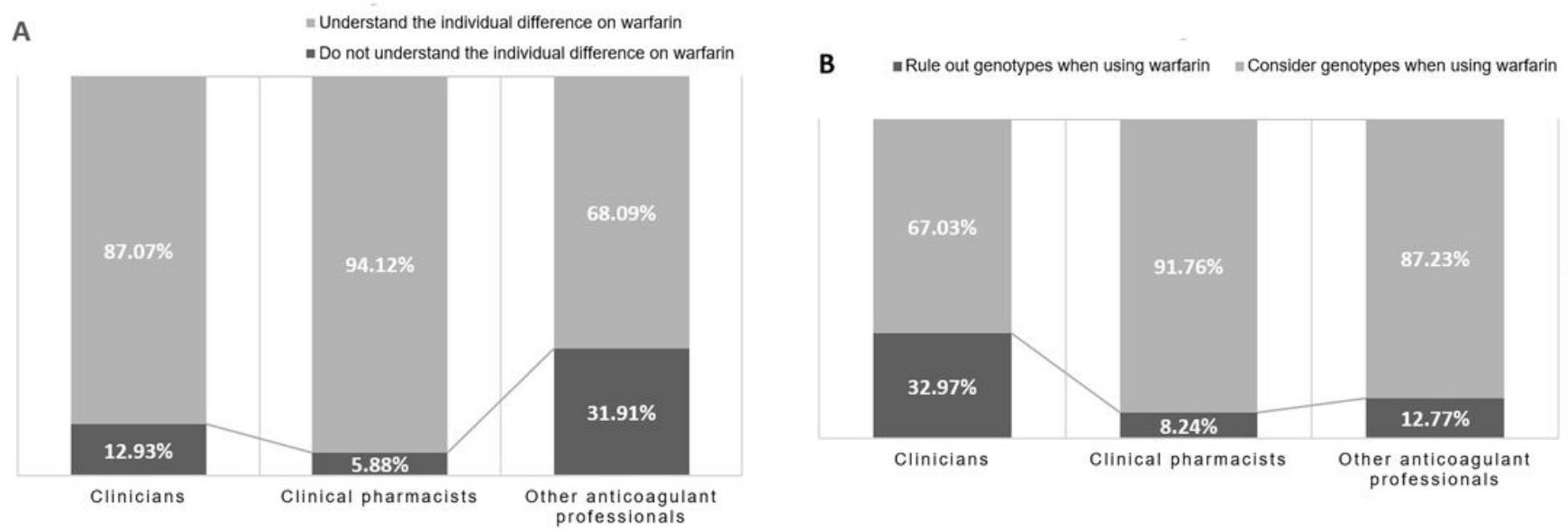
Figure 1

A Knowledges of individual variance on warfarin B Genotypic considerations when using warfarin

\section{Demand for assistant warfarin medication systems}

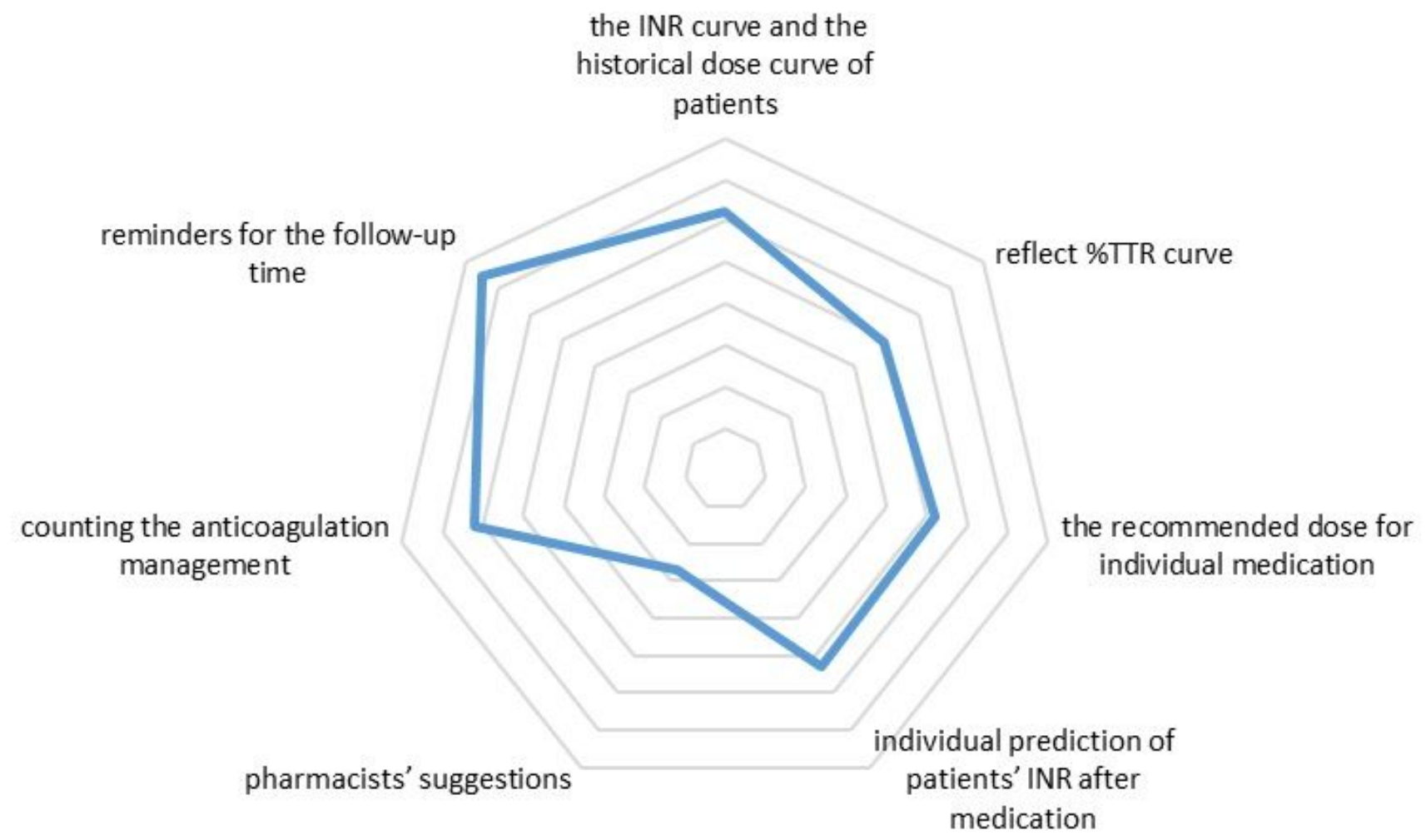

Figure 2

Demand for assistant warfarin medication systems

\section{Supplementary Files}

This is a list of supplementary files associated with this preprint. Click to download.

- Questionnaire.pdf 